Aims. To examine the factors that relate to antipsychotic prescribing in general practices across England and how these relate to cost changes in recent years.

Background. Antipsychotic medications are the first-line pharmacological intervention for severe mental illnesses(SMI) such as schizophrenia and other psychoses, while also being used to relieve distress and treat neuropsychiatric symptoms in dementia.

Since 2014 many antipsychotic agents have moved to generic provision. In 2017_18 supplies of certain generic agents were affected by substantial price increases.

Method. The study examined over time the prescribing volume and prices paid for antipsychotic medication by agent in primary care and considered if price change affected agent selection by prescribers.

The NHS in England/Wales publishes each month the prescribing in general practice by BNF code. This was aggregated for the year 2018_19 using Defined Daily doses (DDD) as published by the World Health Organisation Annual Therapeutic Classification (WHO/ATC) and analysed by delivery method and dose level. Cost of each agent year-on-year was determined.

Monthly prescribing in primary care was consolidated over 5 years (2013-2018) and DDD amount from WHO/ATC for each agent was used to convert the amount to total $\mathrm{DDD} /$ practice.

Result. Description

In 2018_19 there were $10,360,865$ prescriptions containing 136 million DDD with costs of $£ 110$ million at an average cost of $£ 0.81 / \mathrm{DDD}$ issued in primary care. We included 5,750 GP Practices with practice population $>3000$ and with $>30$ people on their SMI register.

Effect of price

In 2017_18 there was a sharp increase in overall prices and they had not reduced to expected levels by the end of the 2018_19 evaluation year. There was a gradual increase in antipsychotic prescribing over 2013-2019 which was not perturbed by the increase in drug price in $2017 / 18$.

Regression

Demographic factors

The strongest positive relation to increased prescribing of antipsychotics came from higher social disadvantage, higher population density(urban), and comorbidities e.g. chronic obstructive pulmonary disease(COPD). Higher \%younger and \%older populations, northerliness and non-white (Black and Minority Ethnic (BME)) ethnicity were all independently associated with less antipsychotic prescribing.

Prescribing Factors

Higher DDD/general practice population was linked with higher \%injectable, higher \%liquid, higher doses/prescription and higher \%zuclopenthixol. Less DDD/population was linked with general practices using higher \%risperidone and higher spending/dose of antipsychotic.

Conclusion. Higher levels of antipsychotic prescribing are driven by social factors/comorbidities. The link with depot medication prescriptions, alludes to the way that antipsychotics can induce receptor supersensitivity with consequent dose escalation.

\section{Different onset patterns of monthly paliperidone palmitate in hospitalised patient diagnosed with schizophrenia}

Javier Herrera-Sánchez ${ }^{*}$, Julia Aznar, Leire Izaguirre and Santiago Ovejero

Hospital Universitario Fundación Jiménez Díaz

${ }^{\star}$ Corresponding author.

doi: 10.1192/bjo.2021.459
Aims. Paliperidone palmitate long-acting injectable (PPLAI) initially requires two loading doses of 150 and $100 \mathrm{mg}$ on days 1 and 8 ( \pm 4 days) intramuscularly. In clinical practice, different PPLAI initiation patterns have been observed. The aim of this study is to describe different PPLAI onset patterns in hospitalised patients diagnosed with schizophrenia.

Method. A naturalistic, transversal, retrospective, descriptive study was carried out. Patients were recruited in the adult inpatient unit of Hospital Universitario Jiménez Díaz (Madrid, Spain) from November 2012 to February 2021. During this period, a total of 357 patients were treated with PPLAI, 172 of them were diagnosed with schizophrenia and, among these, 24 received an atypical onset pattern during hospitalization. Different PPLAI onset patterns, PPLAI dose at discharge and number of days hospitalised were analysed. This study followed the Declaration of Helsinki principles and was approved by the Local Ethics Committee. All participants gave written informed consent.

Result. The sample presents 24 patients (17 men, 7 women) that represents $6.72 \%$ of a global sample, with an average age of 40.21 years (men 35.59 years vs. women 51.43 years). In this study, different PPLAI onset patterns were described: those receiving $150-150 \mathrm{mg}$ represent $25 \%$ of the sample $(n=6)$, as do those receiving $100-75 \mathrm{mg}$, also representing $25 \%$ of the sample $(n=6)$. The rest of the onset patterns were: $150-75 \mathrm{mg}(20.83 \%$, $\mathrm{n}=5), \quad 100-100 \mathrm{mg}(12.5 \%, \mathrm{n}=3), 150-75 \mathrm{mg} \quad(4.16 \%, \mathrm{n}=1)$, $100-50 \mathrm{mg}(4.16 \%, \mathrm{n}=1), 75-100 \mathrm{mg}(4.16 \%, \mathrm{n}=1)$, and $75-75$ $\mathrm{mg}(4.16 \%, \mathrm{n}=1)$. The average hospital stay is 17.88 days. The PPLAI maintenance dose at discharge was $104.17 \mathrm{mg} / \mathrm{month}$. The group of patients who received two doses of $150 \mathrm{mg}$ (150-150 mg) had an average length of stay of 27.67 days compared to the rest of the patients who had an average length of stay of 15.12 days, this difference being statistically significant $(\mathrm{p}=0.010)$. The $150-150 \mathrm{mg}$ group was discharged with a mean maintenance dose of $141.67 \mathrm{mg}$ versus the other patients who needed a mean maintenance dose of $91.18 \mathrm{mg}$, which was also statistically significant $(\mathrm{p}=0.001)$.

Conclusion. The most used pattern of atypical onset of PPLAI in this sample is $150-150 \mathrm{mg}$ and $100-75 \mathrm{mg}$. Patients treated with 150-150 mg loading pattern are hospitalized for a longer period and needed higher maintenance dose at discharge. Further studies are needed.

\section{Adjunct pharmacotherapy for psychotherapy}

Jessica Lochtenberg ${ }^{1 \star}$, Ari Kirshenbaum ${ }^{2}$ and Matthew Johnson ${ }^{3}$ ${ }^{1}$ Royal College of Surgeons in Ireland; ${ }^{2}$ Saint Michael's College and ${ }^{3}$ Johns Hopkins University

${ }^{*}$ Corresponding author.

doi: $10.1192 /$ bjo.2021.460

Aims. A variety of pharmacotherapies have been used to assist the psychotherapy process as "adjunctive therapies." These drugs are used in an acute, targeted fashion, such that they are explicitly delivered in the context of psychotherapy for anxiety, mood and substance-dependence disorders (SUDs). Our narrative review highlights the potential of medically-assisted psychotherapy by outlining the current state of research on few of these medications and describing the basic science that supports their use.

Method. Firstly, we researched an assortment of medications that have been used off-label to enhance psychotherapy, and selected a few that have received the most empirical attention in preclinical and clinical-trial settings. Our review of clinical trials focused on three of the most common psychiatric ailments. For all studies reviewed, we identify the strengths and weaknesses of the data 
supporting the use of the medications for the three aforementioned disorders.

Result. D-cycloserine: accelerates the process of associative emotional learning, enhancing exposure therapy in the treatment of various anxiety disorders, including obsessive-compulsive disorder and posttraumatic stress disorder. Limited studies are available on efficacy in treating SUDs.

Intranasal oxytocin: accelerates memory retrieval-extinction procedures used in posttraumatic stress disorder, and promotes prosocial cognition and behaviour, facilitating a therapeutic alliance. Sufficiently powered studies and safety studies are required before strong conclusions can be made.

Propranolol: interrupts the reconsolidation of memories (leading to maladaptive learned responses) involved in posttraumatic stress disorder during memory-reactivation therapy sessions, but there is little evidence that this drug can be used for depression or SUDs.

Psychedelics: may effect the brain's default mode network, engendering a transformative experience that is often followed by a reduction in psychiatric symptoms. 3,4-methylenedioxymethamphetamine may additionally modulate the amygdala response in a way that allows for reprocessing of traumatic memories, and improves the therapeutic alliance. Anxiety, mood, and SUDs appear to be positively influence by traditional and nontraditional (ketamine) psychedelics.

Conclusion. Although the efficacy of the medically-assisted psychotherapies reviewed is still under investigation, we propose that these novel treatment approaches may be preferred over traditional psychopharmacological treatments due to the presence of fewer chronic side effects, as well less toxicity and abuse potential. Furthermore, these adjunctive pharmacotherapies may help to reinforce the psychotherapeutic alliance and may ultimately yield better long-term treatment outcomes. If at least some of the adjunctive pharmacotherapies outlined in this review are found to be clinically efficacious and safe, patients will benefit from having more treatment options available to them in the future.

\section{Potential drug targets in the kynurenine pathway to treat acute schizophrenia}

Ayush Malhotra ${ }^{1 \star}$ and Paul Manowitz ${ }^{2}$

${ }^{1}$ Rutgers University Graduate School of Biomedical Sciences and

${ }^{2}$ Rutgers Robert Wood Johnson Medical School

${ }^{\star}$ Corresponding author.

doi: 10.1192/bjo.2021.461

Aims. Schizophrenia is a serious developmental psychiatric disorder with a neurodegenerative component that causes marked deterioration in social relationships and ability to work. Present treatments are not satisfactory. Meta-analysis of placebocontrolled studies in acute schizophrenia shows that only a minority of patients have a good response to current antipsychotic medications. Therefore, there is a need for more effective psychopharmacologic treatments for this disorder.

Method. The purpose of this paper is to provide new interpretations of existing data to provide a scaffolding for the development of novel drug targets for the treatment of schizophrenia. The causes of schizophrenia are most likely heterogeneous and involve both genetic and environmental factors. The authors examined a wide range of purported causes of schizophrenia to identify a common biochemical pathway that would contribute to this disorder. This review specifically did not consider pathways that supported the dopamine hypothesis of schizophrenia since historically drugs focused on dopaminergic mechanism, as noted in the aims, have not been successful for many patients with schizophrenia.

Result. Two prominent schizophrenia-associated factors that have been widely studied with significant supporting evidence are stress and inflammation. Stress and inflammation share a common biochemical pathway that converges on the kynurenine pathway of the metabolism of tryptophan, an essential amino acid. At one end of the pathway, recently hospitalized patients with schizophrenia have been found to have low plasma tryptophan levels, whereas chronic schizophrenics have not, suggesting stress- and/or inflammation-induced increased metabolism of tryptophan. At the other end of the pathway, there is increased level of cerebrospinal fluid kynurenic acid in patients with schizophrenia as compared with healthy controls. Salivary kynurenic acid is associated with stress intolerance in schizophrenia. Importantly, natural occurring compounds in this pathway have significant CNS effects that include neurotoxicity and altered neural transmitter behavior.

Conclusion. Stress and inflammation, both associated as causes of schizophrenia, are linked by a common biochemical pathway involving kynurenine. Examination of specific elements of the kynurenine pathway may aid in the identification of drug targets for schizophrenia.

\section{A narrative review of the pharmacological management of psychosis in Alzheimer's disease}

Alexander Matthews ${ }^{1 *}$, Georgina Priest ${ }^{1}$, Sophie Thomas ${ }^{1}$, Hannah White ${ }^{1}$ and Stephen De Souza ${ }^{2}$

${ }^{1}$ Bristol Medical School and ${ }^{2}$ Taunton and Somerset NHS Foundation Trust

${ }^{\star}$ Corresponding author.

doi: $10.1192 /$ bjo.2021.462

Aims. Dementia is estimated to affect 50 million people worldwide, with around $60 \%$ of these cases attributable to Alzheimer's disease (AD). One of the common behavioural and psychological symptoms associated with $\mathrm{AD}$ is psychosis. Psychosis, experiencing delusions or hallucinations, can be one of the most distressing ordeals for patients with $\mathrm{AD}$, as well as those around them. Effectively managing these symptoms can lead to a vast improvement in life quality. Currently, there are no medications specifically licensed in the UK for the treatment of psychosis in AD. To help guide clinical practice, we reviewed the evidence underpinning the pharmacological treatment of psychosis in $\mathrm{AD}$. The aim of the study was to positively influence clinical practice and thereby improve the life quality of this patient group.

Method. An advanced PubMed search was used to identify studies which investigated the pharmacological treatments for acute psychosis in people with AD. Papers included were double blind, placebo controlled, randomised controlled trials specifically for $\mathrm{AD}$ dementia. Papers must have reported their findings using a specific psychosis subscale (PS); examples being "Behavioural Pathology in AD" (BEHAVE-AD-PS), "Brief Psychiatric Rating Scale" (BPRS-PS), and "Neuropsychiatric Inventory - Nursing Home Version" (NPI-NH-PS). Populations of both outpatients and residential patients were accepted. 14 papers, comprising some 3237 patients, were included and critically analysed in the final review.

Result. Risperidone (BEHAVE-AD-PS: -1.3 [p=0.004] \& -1.9 [ $p=0.039]$; BPRS-PS: $-0.5[\mathrm{p}=0.08]$ ) and aripiprazole (NPINH-PS: $-1.0[p=0.169] \&-1.8[p=0.013])$ successfully reduced psychosis symptoms in patient populations. However, these medications were associated with a statistical increase in severe adverse 